\title{
Physical human-robot interaction for clinical care in infectious environments
}

\author{
The COVID-19 pandemic has highlighted key challenges for patient care and health provider safety. Adaptable \\ robotic systems, with enhanced sensing, manipulation and autonomy capabilities could help address these \\ challenges in future infectious disease outbreaks.
}

\author{
Hao Su, Antonio Di Lallo, Robin R. Murphy, Russell H. Taylor, Brian T. Garibaldi and Axel Krieger
}

( uring the COVID-19 pandemic, the surge of patients who require hospitalization and the need to physically isolate them to prevent disease transmission have created unique challenges for clinical care. An infectious disease event such as COVID-19 disrupts the ordinary healthcare workflow by placing an unsustainable burden on a small set of care pathways. Additionally, the provision of care must take place with protection in place for both caregiver and patient. Each direct contact with infected patients is a source of infection for healthcare workers, and the infection of a caregiver translates into a reduced hospital workforce and an increased workload for the other colleagues. While healthcare workers are less than $3 \%$ of the population in the majority of countries, they represent around 14\% of COVID-19 cases reported to the World Health Organization ${ }^{1}$. According to a recent study collecting data from 37 nations, nearly 300,000 infections and over 2,500 deaths have been reported among healthcare workers as of August $2020^{2}$. These difficulties have placed a tremendous burden on an already limited workforce and emphasize the need to protect caregivers.

Another effect of the pandemic has been a large-scale deferral of elective surgical procedures (nearly five million in the first three months ${ }^{3}$ ) to mitigate the risk of virus transmission. Even though they involve non-emergency care, these surgeries are not optional, and the delays may have long-term consequences on the well-being of patients. Moreover, they have tremendous implications on the economic and financial affairs of healthcare systems and their satellite activities. The American Hospital Association (AHA) estimates an average financial impact of US $\$ 50.7$ billion per month in losses for America's hospitals and health systems. Innovative technologies can provide a valuable aid to tackle these difficulties. Telemedicine has substantially reduced in-person healthcare visits and is

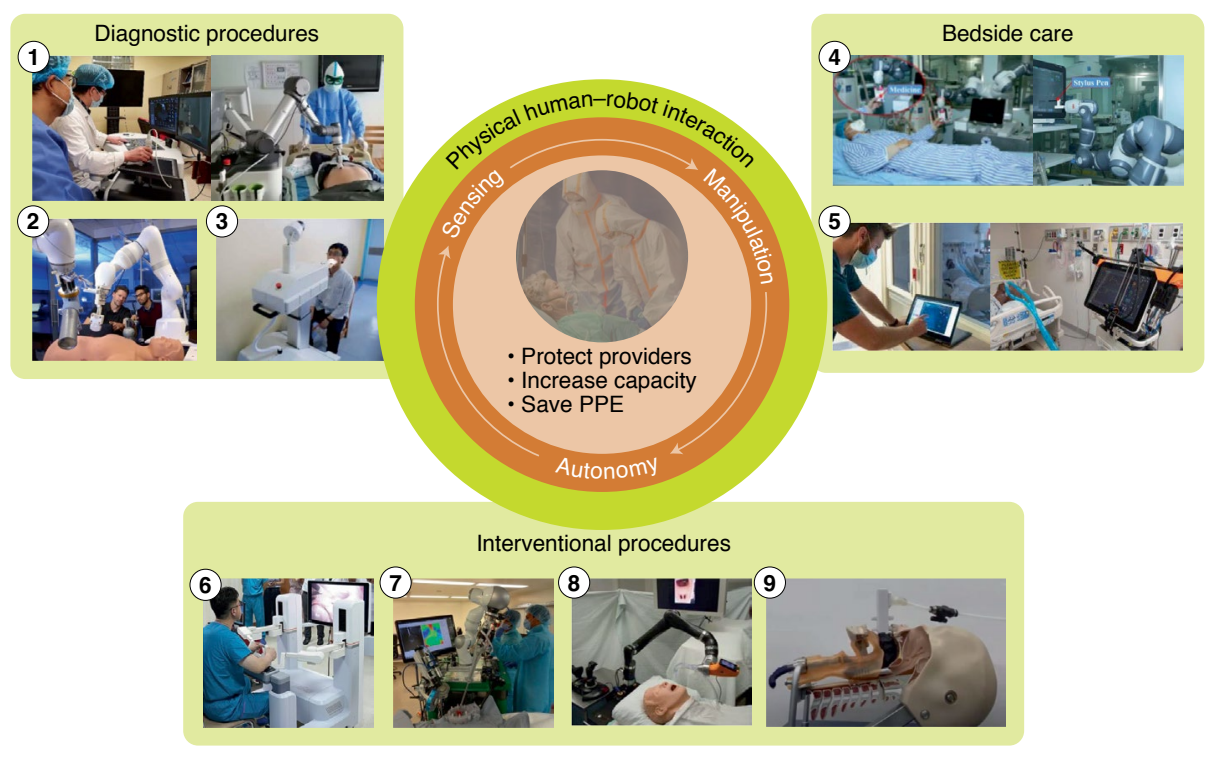

Fig: 1 | Physical human-robot interaction. To tackle clinical challenges in acute care of infectious diseases, convergent research areas of physical human-robot partnerships, including sensing, manipulation and autonomy, will enable highly flexible and versatile medical robots, with enhanced capabilities to feel, touch and decide. The robots featured in the figure: (1) robot-assisted ultrasound imaging for remote examination of lungs and hearth in patients with COVID-197; (2) robot-assisted remote trauma assessment, including ultrasound imaging to evaluate the severity of lung involvement in patients with COVID-1920; (3) semi-automatic oropharyngeal-swab sampling used for COVID-19 testing ${ }^{6}$; (4) nursing assistance provided through a teleoperated robotic platform ${ }^{10}$; (5) medical staff using a robot to remotely control ventilators in intensive care units of patients with COVID-1911; (6) robot-assisted surgery, where $5 \mathrm{G}$ technology was exploited to perform four ultra-remote laparoscopic procedures $^{16} ;(7)$ supervised autonomous soft-tissue surgery successfully tested on porcine bowel anastomosis $^{18} ;$ (8) robot-assisted endotracheal intubation ${ }^{12} ;(9)$ endotracheal intubation performed via a soft robot that can grow into multiple branches through the respiratory airways ${ }^{13}$. Figure reproduced with permission from ref. ${ }^{7}$, Elsevier (1); ref. ${ }^{6}$, ERS (3); ref. ${ }^{10}$, Springer Nature Ltd (4); ref. ${ }^{16}$, Springer Nature Ltd (6); ref. 12, Elsevier (8); Hawkes Lab /David Haggerty and Luis Ramirez (9).

a key strategy for healthcare surge control ${ }^{4}$. However, current telesystems provide mostly communication support, while physical interactions required for diagnostic procedures, interventional procedures and bedside care (Fig. 1) still largely rely on in-person visits. Separating providers from patients using physical dividers with gloved ports is compatible with only limited interactions. Personal protective equipment
(PPE) allows providers to perform in-person tasks safely but increases the difficulty of many tasks, is uncomfortable for prolonged periods and is in limited supply. Physical human-robot interactions ${ }^{5}$ offer promising solutions to these challenges.

There are three major areas where robotics can enhance patient care and provider safety: (1) minimizing contact of caregivers with infected patients to reduce 
infection transmission; (2) increasing capacity and efficiency of clinical providers so they can focus on important tasks; (3) reducing the supply requirements for $\mathrm{PPE}$ by limiting the number of times providers enter an isolation area. This Comment summarizes the state of the art in medical robotics to address these challenges. It suggests how the latest technologies can be leveraged to enable the development of highly flexible and versatile robots for future infectious disease crises. Overall, advances in robot sensing, manipulation and autonomy are required to improve robots' capabilities to feel, touch and decide.

\section{Robot sensing and manipulation}

Sensing is critical to planning and executing safe physical human-robot interactions. Massive testing is fundamental to isolate infected subjects and hamper the spread of the virus. Rapid assessment and diagnosis with swab robots ${ }^{6}$ could reinforce testing capacity with the reduced engagement of healthcare workers.

An important clinical challenge during COVID-19 is to assess and monitor disease severity. Computed tomography (CT) has the greatest sensitivity to identify lung pathology, but requires transport to a radiology suite, with increased risk of infectious exposure and prolonged downtime to disinfect equipment. Radiographs can be performed more rapidly at the bedside but have poor sensitivity compared to CT. Point-of-care ultrasound (POCUS) is a promising modality to assess the presence and severity of lung pathology in COVID-19. It provides real-time actionable imaging data, corresponds well to findings on CT scan, and limits potential exposures only to necessary personnel. However, POCUS requires more advanced training to perform and interpret and is highly operator dependent. Robot-assisted remote ultrasound systems successfully imaged patients with COVID-197, but required more highly trained personnel and substantially longer procedure times. To enable safe imaging by untrained providers, improvements are needed in sensing and autonomy, especially in fusing multimodal sensors from ultrasound, cameras and interaction forces.

Visual sensing combined with tactile feedback can incredibly enhance the navigation and operation capabilities of robots. The development of soft sensors, along with advances in haptics, paves the way towards artificial skins to give robots a distributed sense of touch ${ }^{8}$. Future breakthroughs may come from the fusion of multiple sensing modalities (for example, pressure, roughness, temperature), from the integration of sensors and actuators, and from the development of control strategies to turn perception into reactive behaviours.

Another key clinical challenge is the ability to perform a wide variety of manipulation tasks, such as swab sampling, monitoring and managing a ventilator, adjusting infusion pumps, changing intravenous fluid bags, and physically moving patients. Therefore, dexterous manipulation of diverse medical tools is a key requirement for robots ${ }^{9}$. Collaborative robots have already demonstrated impactful functionalities ${ }^{10}$, and advances in soft robotics offer new enabling solutions. Inherent adaptability of compliant materials, multiple grasping modalities, and stiffness modulation can be used in concert to enable a single robot to perform both delicate and strong manipulation tasks. Robots have also been designed to remotely operate ventilators from outside an intensive care unit room to prevent exposure risk for healthcare workers ${ }^{11}$. Increasing the manipulation dexterity and ease of end-user programming of these systems is needed to make such solutions quickly deployable for a multitude of equipment and control tasks.

About $8-11 \%$ of patients infected with COVID-19 develop hypoxemic respiratory failure and require mechanical ventilation. A crucial step to assist a patient in respiratory failure is endotracheal intubation. This procedure relies heavily on the skill of experienced clinicians and may lead to aerosolization of virus particles. Existing robotic systems ${ }^{12}$ can potentially be adopted to facilitate intubation and reduce the risk of healthcare worker infection. These systems need to be failsafe and manoeuvrable to be effectively deployed. One promising approach is a vine-inspired soft robot $^{13}$ that creates a conduit to the lungs with the ability to grow into airways, using multiple branches to accommodate different morphologies. The low cost and manufacturability of many soft robots can make the production of disposable devices convenient compared to the disinfection of their reusable counterparts.

\section{Increased autonomy}

Increasing the autonomy of robots can enhance the efficiency of physical humanrobot interactions and augment the capacity of healthcare systems. Learning strategies have already enabled improved performance in robot grasping tasks, with reliability greater than $95 \%$ at a rate of more than 300 mean picks per hour ${ }^{14}$. Instead of requiring a healthcare worker to direct every action of a robot, autonomous robots will be able to perform tasks with minimal or even without direct supervision thus enabling providers to focus on critical aspects of patient care and supervise several robots. Delegation to a robot typically raises questions of (1) how can the robot's task be specified clearly enough, so the robot knows exactly what to do; (2) how can one ensure that the robot will perform the specified task safely and correctly; and (3) how to manage unexpected events and uncertainty. A recent classification distinguished development in autonomous surgery ${ }^{15}$ between level 0 (teleoperation) $)^{16}$ and level 5 (full autonomy) ${ }^{17}$, with few systems reaching beyond level 2 for task autonomy, such as that for autonomous suturing ${ }^{18}$. Autonomous physical interactions with bony rigid tissue anatomy based on preoperative model acquisition and computer-assisted surgical planning have already improved surgical outcomes. In contrast, interaction with soft tissues poses prohibitive challenges due to unpredictable, elastic and plastic deformations. Patient to patient variations impose another level of challenge on automating physical robot interactions. These challenges suggest the most realistic approach for healthcare robots in the near future is shared autonomy, which combines the knowledge of medical experts with the capabilities of robots.

Future robots will play a much greater role in clinical care for infectious diseases. Capability, mobilization and infectious disease environment-specific barriers ${ }^{19}$ must be overcome to achieve dexterous, rapidly deployable, and intelligent systems, with increased autonomy and enhanced capabilities of physical interaction. Following this path, it is envisioned that robotic technologies will bring enhanced solutions to the side of clinicians, providing healthcare workers with tools to safely, remotely and effectively treat patients during the next infectious disease outbreak.

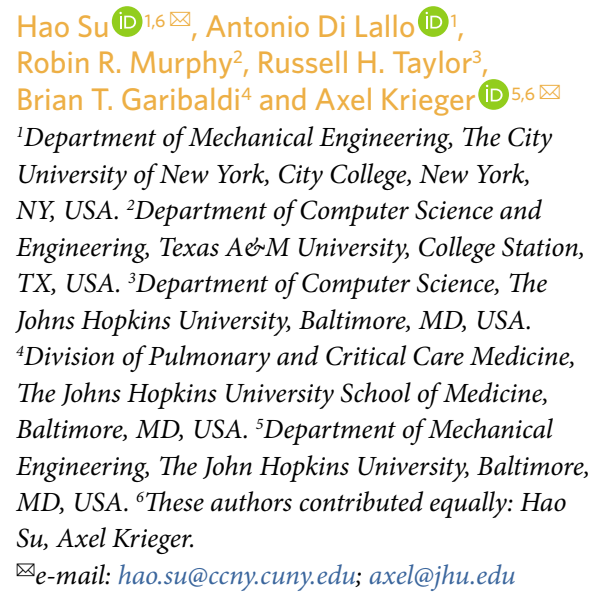

Published online: 18 March 2021

https://doi.org/10.1038/s42256-021-00324-z 


\section{References}

1. Fraher, E. P. et al. New Engl. J. Med. 382, 2181-2183 (2020).

2. Erdem, H. \& Lucey, D. R. Int. J. Infect. Dis. 102, 239-241 (2021)

3. Fu, S. J., George, E. L., Maggio, P. M., Hawn, M. \& Nazerali, R. Ann. Surg. 272, e79-e80 (2020).

4. Hollander, J. E. \& Carr, B. G. New Engl. J. Med. 382, 1679-1681 (2020).

5. Nat. Mach. Intell. 2, 553-553 (2020).

6. Li, S.-Q. et al. Eur. Respir. J. 56, 2001912 (2020).

7. Ye, R. et al. Chest 159, 270-281 (2021).

8. Shih, B. et al. Sci. Robot. 5, eaaz9239 (2020).
9. Hauser, K. \& Shaw, R. IEEE Spectrum https://spectrum. ieee.org/automaton/robotics/medical-robots/ medical-robots-future-outbreak-response (2020).

10. Yang, G. et al. Chin. J. Mech. Eng. 33, 47 (2020).

11. Vagvolgyi, B. P. et al. Preprint at https://arxiv.org/abs/2010.05247 (2020).

12. Hemmerling, T. et al. Brit. J. Anaesth. 108, 1011-1016 (2012).

13. Hawkes, E. W., Blumenschein, L. H., Greer, J. D. \& Okamura, A. M. Sci. Robot. 2, eaan3028 (2017).

14. Mahler, J. et al. Sci. Robot. 4, eaau4984 (2019)

15. Zemmar, A., Lozano, A. M. \& Nelson, B. J. Nat. Mach. Intell. 2 566-572 (2020)
16. Zheng, J. et al. Surg. Endosc. 34, 5172-5180 (2020).

17. Yang, G.-Z. et al. Sci. Robot. 2, eaam8638 (2017).

18. Shademan, A. et al. Sci. Trans. Med. 8, 337ra64 (2016)

19. Hager, G., Kumar, V., Murphy, R., Rus, D. \& Taylor, R. Preprint at https://arxiv.org/abs/2010.09909 (2020).

20. Mathur, B. et al. in 2019 IEEE 19th International Conference on

Bioinformatics and Bioengineering 649-656 (IEEE, 2019).

Competing interests

The authors declare no competing interests. 